\title{
Conversation-analysis (CA) as a tool for exploring interaction in an online video-conferencing based support service.
}

\begin{abstract}
Purpose

Around $60 \%$ of people with dementia in the UK live at home. The experience of caring for a family member with dementia can be rewarding and positive, but it can also be significantly stressful. Current healthcare policy is encouraging greater provision to support family carers. Along with respite-care, day-care and support group-based initiatives, there has also been a focus on developing dementia-specific communication training.
\end{abstract}

\section{Approach}

We outline a new initiative 'Empowered Carers' which is being piloted in the North of England. Empowered Carers is an online support and communication training service for family carers who are caring for someone with dementia at home. It utilises online video conference-calling technology to connect carers with support workers, and also allows for simultaneous interactions involving other family members. A central tenet of the approach is a theoretically grounded support model, based on the concept of mentalisation.

\section{Findings}

We describe the background to Empowered Carers, and how a conventional evaluation strategy for the initiative is being used alongside a socio-linguistic approach (Conversation Analysis - CA). This aims to provide empirical evidence about how the assimilation of mentalisation is reflected in the structuring of speech patterns in carers during support sessions.

Value

We explain the CA method, how it has been applied to similar talk-based therapeutic settings, and why its ability to explore sequential linguistic patterns across extremely large data-sets is particularly suited to studying interaction in emerging online arenas.

Keywords:

Conversation Analysis (CA); carer support; Video-conferencing; real-time online support services; mentalisation; family carers; dementia.

Article Classification:

Case study 


\section{Background}

Nearly two thirds of people with dementia in the UK live at home (Dept of Health, 2013). But while the experience of caring for a family member with dementia can be rewarding and positive, for the majority of carers it can also be significantly stressful (de Vugt \& Verhey, 2013; Robertson et al, 2007). Family carers of people with dementia are often required to provide high levels of assistance, commit a large proportion of their time, and manage a range of challenging behaviours in the people they care for. These can range from repetitive speech, wandering, and sleep disturbances, through to refusal of care, argumentation and violent outbursts (Gitlin et al 2010; Schulz \& Martire, 2004). If unaddressed, these behaviours have been shown to accelerate disease progression, worsen functional decline, and result in earlier nursing home placement (Gitlin et al, 2012). They will also have a broader impact on the quality of life of both the person with dementia and the family carer.

For many family carers the perception that they are not properly equipped to deal with what may be a new and unexpected role can also be a considerable strain (Fauth et al, 2006; Ballard et al, 2000), and it can be difficult to reconcile the role of caregiver with that of spouse, child or sibling (Wenger, 1997). Social isolation for family carers can also be a problem because, although social attitudes towards dementia are changing, (Berges et al, 2003) the condition is still widely associated with negative stereotypes such as the fear of losing one's independence and being a burden to others (Oconnor et al, 2010). As the family member with dementia becomes progressively more dependent and the demands placed upon the caregiver's time and emotional resources become more consuming, family tensions arise that can be difficult to talk about (Kramer et al, 2006), not least because of the damaging and unpredictable effects that dementia can have on communication and cognitive processes - even in its early phases.

In the light of ongoing dementia-focused policy initiatives (Dept. of Health, 2009; 2015; 2016) support for family carers is becoming more of a priority, and there has been considerable interest in developing communication-focused training-interventions for carers of people living with dementia.

Many of these have sought to take advantage of the possibilities offered by online and web-based delivery. McKechnie et al (2014), for example, examined computer-mediated support services for family carers and found that they often reduced levels of carers carer burden/stress and depression. Levels of anxiety were also reportedly reduced, and appreciation of the positive aspects of caring were increased. However, in a recent systematic review of the field, Morris et al (2017) found that while many such interventions are successful in terms of improving carers' knowledge of communication skills and strategies, only a minority actually have an impact on their levels of wellbeing. It has been suggested that one reason for this is that many interventions lack, or fail to adequately integrate, an appropriate theoretical model (Morris et al, 2018), and that this shortcoming can prevent an understanding of the mechanisms through which beneficial effects are realised (Elvish et al, 2013; Popay et al, 2006).

In this article we use the example of a newly developed online carer-support intervention - which importantly, does have a secure theoretical basis - to illustrate how sociolinguistic approaches (specifically conversation analysis - CA) can be used to better understand connections that might exist between the language and interactional characteristics of people who have internalised a given system of support, and those who have not, or who are at various levels of adjustment to it. We outline how CA works, and how its 
ability to track linguistic patterning across extremely large data-sets is particularly suited to studying the extensive corpuses of naturalistic video data that are becoming available in emerging online support arenas.

\section{Empowered Carers}

Empowered Carers is a carer support initiative based on the well-established New York University Caregiver Intervention (Gaugler et al, 2011; Mittelman, et al, 2004). This approach has been adapted widely and generally consists of an initial course of 4 or more sessions of individual and family counselling, followed by additional on-demand telephone support. The programme may also include access to a support group or informal support network. In the case of Empowered Carers, the intervention offers 2 initial face-to-face sessions for the main carer and 4 sessions for other family members. Ongoing support is then offered via secure online video-conferencing software which can be freely downloaded and accessed on any internet enabled device (Zoom Video Communications, 2018). The dementia communication approach offered by the service is based on the concept of Mentalization (Allen et al, 2008). This is ' . . the imaginative mental activity that enables us to perceive and interpret human behaviour in terms of intentional mental states.' (Fonagy \& Luyten, 2009, p. 1357). Essentially, support is offered that helps carers to become more reflective, more attuned to their own state of mind, and more able to recognise their emotional response to the person with dementia. In practical terms, although carers using Empowered Carers do not necessarily need to engage with it at a theoretical level, it will give them the emotional resources they need to stay connected, handle emotional stress and navigate their day when they encounter challenging situations.

Empowered Carers is currently a pilot initiative funded by a local NHS Clinical Commissioning Group (see acknowledgements). And as such, its effectiveness will be carefully evaluated using a variety of established measures. These will assess outcomes including levels of care-giver depression and the quality of life and social health of the person being cared for. In addition to a standard service evaluation however, the initiative will also be providing data for a socio-linguistic study which will use Conversation Analysis (CA) to conduct an in-depth micro-analysis of the family carer / support worker interactions that take place on the platform.

\section{Using conversation analysis (CA)}

Conversation analysis is well established as a highly effective method for investigating interaction in a wide variety of settings. The approach is largely concerned with the analysis of the verbal communicative practices that people routinely use when they interact with one another. Utilising video and audio recordings of naturally occurring interaction, and a highly-detailed method of transcription which captures the minutiae of speech and aspects of non-verbal behaviour, it provides an analytical method that can expose the underlying 'rules' that govern how activities are composed and organised. (Drew et al, 2001). From a CA perspective, the study of therapeutic interaction - and we would argue that the carer / support worker interactions we are concerned with in Empowered Carers can essentially be classed as such - aims to illustrate at an empirical level how a given approach actually 'gets done' (Georgaca \& Avdi, 2009). It can help to uncover and describe skills and competences that therapists or support workers themselves might be using at an unconscious level, and complement and enhance professional knowledge (Peräkylä \& Vehviläinen 2003; Peräkylä et al. 2008; Silverman 1997). Similarly, it can show how patients, clients, or in this 
case family carers, are adapting their interactions as they incorporate the theoretical model that underpins their training.

\section{CA data example}

One way to illustrate CA is to present an example of how it has been used in a broadly similar context, and describe an interactional effect that is likely to be prominent in the Empowered Carers encounters we are concerned with. In their CA based study of telephone delivered cognitive behavioural therapy sessions (TCBT), Chatwin et al (2018) observed that occurrences of extended silence, which in everyday conversation might be regarded as interactionally 'troublesome', are in fact often oriented to by client and therapist as non-problematic, even in the context of phone interactions when both parties are unable to access any visual cues.

By analysing a large corpus of sequential T-CBT sessions it was observed that it is often the issue of how parties switch between and signal orientation to different meanings for their silence that can create interactional misalignment, rather than the silence itself. Across several sessions, clients appeared to become more adept at recognising the interactional implications of different 'types' of silence by judging which phase of a consultation they were situated in, and what activity they were engaged in - summarising, reflecting on issues of concern to them, reporting on how they had been feeling and so on.

Extracts 1 and 2 (below) are from the T-CBT study and illustrate how effects such as instances of extended silence can impact on interaction in predictable ways. They are also an example of how the detailed transcription method used in CA (see box 1 for transcription conventions) conveys information not only about what is actually said, but also about the relative temporal positioning of the turns at talk, the minutiae of speech, and in the case of video-recorded interaction, aspects of non-verbal behaviour. (See box 1 for the meaning of the punctuation symbols used in CA transcriptions.)

\section{Extract 1}

1 T: So do you think your mood's stabilised,

2 do you think now?

$3 \quad(1.5)$

$4 \quad$ C: $\quad$ Ye::a?

$5 \quad(1.0)$

$6 \quad \mathrm{~T}: \quad$ Yea?

$7 \quad$ C: $\quad$ Just er: more positive thinking really.

$8 \quad \mathrm{~T}: \quad$ The more positive thinking. 


\author{
Extract 2 \\ $1 \quad \mathrm{~T}: \quad \ldots$ sometimes you'll stop, but sometimes \\ 2 you'll carry on \\ 3 working through the pain. Have I got \\ $4 \quad$ that right? \\ $5 \quad$ C: $\quad$ Yea. .hh but that's just cos it's not \\ $6 \quad$ that strong, $\mathrm{y}^{\prime}$ know or else I \\ 7 feel real sorry for myself. . .
}

These two basic examples illustrate the influence that the sequential location and duration of pauses and silences can have within ongoing interaction. Although in many counselling and therapeutic settings fairly extended periods of silence are very common (Chatwin et al, 2018), there is a basic CA tenet that in everyday conversation, pauses or silences that are over about 1 second duration can indicate some form of interactional 'trouble'. (Jefferson, 1989). In these extracts it can be seen that the therapists and clients are orienting to this 'everyday' mode of interaction.

In extract 1, the therapist makes an enquiry about the clients mood (lines 1 and 2 ), and the client confirms that it has stabilised (line 4). Had this been a verbatim transcript of the encounter, little more information could be inferred. However, from a CA perspective, a potential misalignment between what is being claimed by the client, and what is actually conveyed in the structure of her talk is evident. Even though her reply is affirmative, this comes after an extended ( 1.5 second) pause (line 3 ). It is also combined with the elongated delivery and upward intonation of her 'ye::a?', indicated in the transcript by the three colons and questionmark (line 4). These micro-interactional elements conspire to convey that, while it cannot be assumed that what she says is actually untrue, she may well have some reservations or concerns about it. The therapist appears to pick up on this because she also leaves an extended pause before her next turn (line 5), and then uses a rising intonation (again, indicated in the transcript by a question-mark) to prompt the client for more details ('yea?' - line 6).

On the other hand, extract 2 is far less ambiguous and illustrates another common CA convention; that truly affiliative or confirmatory responses usually come immediately after an enquiry - often overlapping slightly with the last syllables of the previous turn. Schegloff (2000) calls this type of response a 'smooth transition.' Here, the therapist asks the patient to confirm that a summary she has just given is accurate. The patient's affirmative reply (line 5) comes immediately after the therapist's question, and conveys an essentially nonproblematic response - although it could be argued that in this case the rest of what the client says suggests there may be other issues, tangentially related to her pain, that need addressing. 
These short examples illustrate how CA can describe individual micro-interactional conventions at work in ongoing talk. Importantly however, if large scale longitudinal data-sets are available, including recordings made of consecutive therapeutic encounters or support sessions, it can also provide a bridge between the theoretical assumptions that underlie a particular therapeutic modality, and how these are actually reflected in the structure of the interaction between therapist or support worker and their client. A key feature of the CA approach is that unlike many of the narrative or thematic based methods which are routinely used to analyse therapeutic interaction, it is one of the few naturalistic qualitative methodologies which can work effectively with really large sets of data (i.e. extensive collections of instances within multiple interactions where a given phenomena occurs). This is important in terms of objectivity and generalisation because the influence of individual participants communication styles, or their particular psychological disposition is effectively removed (Chatwin, 2014; Drew et al, 2001).

\section{What CA can tell us?}

In terms of the mentalisation focused support that we are concerned with, CA can provide empirical data on the underlying conversational features that are particular to people who are beginning to engage in more effective dementia communication. For example, although the practical mentalisation processes that underly the Empowered Carers intervention are necessarily broad; the development of behavioural coping strategies; focused self-reflection and so on, their ultimate goal is still essentially interactional. The family carer and wider family network are being helped to understand and manage their emotionally influenced behavioural responses so they can communicate more effectively with the person they are caring for.

From a CA perspective, the success or otherwise of this will be evident in the construction of their talk. Aside from the higher-level elements of meaning, context, and content of what they say, there will also be significant (and largely unconscious) micro-interactional effects that underpin these. These might include changes in the relative timing of utterances as they become more familiar with the mechanics of their training; changes in the use and sequential positioning of certain idiomatic constructions within their talk; or perhaps modifications in the frequency and positioning of instances of overlapping speech. There may be any number of micro-effects that could indicate that a certain interactional perspective has been achieved because, as we showed with extracts 1 and 2, even something as simple as a variation in the duration or sequential position of a silence can convey multiple meanings.

This is where CA method can be useful, because when applied to a complete data-set (for example, the several hundred hours of carer/support worker interactions that will potentially be available from Empowered Carers) it can isolate and describe the basic micro-interactional features that develop in the talkprocesses of someone undertaking a particular type of communication training. Then, once these underlying features are identified it can be used in a more focused way to track how assimilation is proceeding in individual cases. This may be where it is of more practical relevance - particularly in terms of evaluating the effectiveness or otherwise of individual carer/support worker encounters that occur on the videoconferencing platform, and judging degrees of progress. Evaluations based on detailed longitudinal microinteractional data are likely to be far more robust that those based, for example, on self-reported satisfaction scales. 
Other CA studies in this and related areas such as counselling and psychotherapy have isolated features including the role of idiomatic expressions in interactions between mental health professionals and their clients (Antaki, 2007), and particular micro-interactional effects, including reformulations, pausing, and abrupt changes of topic, that might indicate a breakdown of empathy between client and therapist (Chatwin et al, 2007; Wynn \& Wynn, 2006). Of direct relevance here, Henrich et al (2018) recently used CA to examine how clients responded to invitations to mentalise in psychotherapy sessions, and suggest that seen from a micro-interactional perspective, the ability to mentalise is not only dependent on a degree of innate ability, but also on how clients are able to use the linguistic resources available to them. In terms of the mentalisation based support encounters occurring with Empowered Carers, we expect to find many of the features outlined in other studies. However, how they mesh, contextualise and become enacted within the additional dimension of a multi-party video conferencing platform is yet to be seen.

\section{Conclusions}

Against the backdrop of ever-greater online integration in healthcare and support services, and the growth of real-time interactive interventions, there is a strong argument for these new therapeutic arenas to receive the same level of micro-interactional analysis that has been applied to most other medical and support settings. In this article we have we have explored how CA can offer insights into how users engage with online interventions, and how it can reveal how those providing support adapt their therapeutic approach to work more effectively in the online context. We have also outlined how CA can provide a better understand of the connections that might exist between the language and interactional characteristics of people who have internalised a given system of support, and those who might be at various levels of adjustment to it. Online and web-based services are becoming an integral part of UK healthcare delivery. For a small but significant proportion of people this shift may be difficult to adapt to, and it could be argued that for some already marginalised groups (for example, those who do not have access to a computer or smartphone, or who may choose not to engage with them) it may actually be making healthcare more inaccessible. However, at a broader level, the potential 'enabling' qualities of innovative services such as Empowered Carers - particularly in terms of making access to support more convenient and direct for a majority of users - would appear to outweigh this.

\section{Acknowledgements}

[Funder details removed for blind review] 


\section{Box 1}

\section{CA transcription symbols}

This is a simplified list of symbols that are used in the transcription of recorded data. In CA, punctuation such as full stops, commas and question marks etc. are used to denote the characteristics of ongoing speech and do not necessarily maintain a conventional grammatical function.

oxo $\quad$ - degree signs indicate speech that is quiet relative to the surrounding talk.

LOUD - capital letters indicate speech which is louder relative to the surrounding talk.

text - underlining indicates emphasis on a word (not necessarily a rise in volume).

- full stops are used to indicating a falling intonation.

- commas indicate continuing intonation.

.h $\quad$ - indicates an in breath.

h. - indicates an out breath

(0.5) - numbers within brackets indicate timings in whole and tenths of a second.

(.) - a full stop within brackets indicates a 'micro pause' of less than 2/10ths of a second.

[ - square brackets are used to denote the points at which speech overlaps.

::: $\quad$ - one or more colons after a word indicate an extended sound (for example, an 'Er' sound that is drawn out might be written: 'Er: : :' The more colons the longer the sound. 


\section{References}

Allen, J.G., Fonagy, P. and Bateman, A.W., (2008). Mentalizing in clinical practice. American Psychiatric Pub.

Ballard C, Lowery K, Powell I et al. (2000) Impact of behavioral and psychological symptoms of dementia on caregivers. Int Psychogeriatr 12:93-105.

Brodaty, H., Thomson, C., Thompson, C. and Fine, M., (2005) Why caregivers of people with dementia and memory loss don't use services. International journal of geriatric psychiatry, 20(6), pp.537-546.

Burgess, L., Page, S. and Hardman, P., (2003). Changing attitudes in dementia care and the role of nurses. Nursing times, 99(38), pp.18-19.

Chatwin, J., Bee, P., Macfarlane, G.J. and Lovell, K., (2018). Observations on silence in telephone delivered cognitive behavioural therapy (T-CBT). Journal of Applied Linguistics \& Professional Practice, 11(1).

Chatwin J (2014) Conversation analysis as a method for investigating interaction in care home environments. Dementia 13. 6: 737-746

Chatwin J, Watt I, Collins S \& Field R (2007) A feeling of equality: some interactional features that build rapport and mutuality in a clinical encounter. In: Collins. S (Ed) Healthcare Communication and Participation in Action. Open University Press

Department of Health, (2013). Dementia: a state of the nation report on dementia care and support in England.

Department of Health. (2012). Prime Minister's Challenge on Dementia: Delivering Major Improvements in Dementia Care by 2015. London. Crown Copyright.

de Vugt, M.E. and Verhey, F.R., (2013). The impact of early dementia diagnosis and intervention on informal caregivers. Progress in neurobiology, 110, pp.54-62.

Elvish R, Leaver S, Johnstone J, Cawley R \& Keady J (2013) Psychological interventions for carers of people with dementia: a systematic review of quantitative and qualitative evidence. Counselling and Psychotherapy Research 13(2) 106-125.

Fonagy P \& Luyten P (2009) A developmental, mentalization-based approach to the understanding of and treatment of borderline personality disorder. Development and Psychopathology. 21(4) 1355-1381. 
Fauth EB, Zarit SH, Femia EE et al. (2006) Behavioral and psychological symptoms of dementia and caregivers' stress appraisals: Intra-individual stability and change over short-term observations. Aging Ment Health;10:563-573.

Georgaca, E. and Avdi, E. (2009) Evaluating the talking cure: The contribution of narrative, discourse, and conversation analysis to psychotherapy assessment. Qualitative Research in Psychology 6 (3): 233-247.

Gitlin, L.N., Winter, L., Dennis, M.P., Hodgson, N. and Hauck, W.W., (2010). Targeting and managing behavioral symptoms in individuals with dementia: a randomized trial of a nonpharmacological intervention. Journal of the American Geriatrics Society, 58(8), pp.1465-1474.

Gitlin, L.N., Kales, H.C. and Lyketsos, C.G., (2012). Nonpharmacologic management of behavioral symptoms in dementia. Jama, 308(19), pp.2020-2029.

Gaugler, J., Roth, D.L., Haley, W.E. and Mittelman, M.S., (2011). Modeling trajectories and transitions: results from the New York University caregiver intervention. Nursing research, 60 (3 Suppl), pp. S28.

McKechnie, V., Barker, C. and Stott, J., 2014. Effectiveness of computer-mediated interventions for informal carers of people with dementia-a systematic review. International Psychogeriatrics, 26(10), pp.1619-1637.

Morris L, Horne M, McEvoy P \& Williams T (2017). Communication training interventions for family and professional carers of people living with dementia: a systematic review of effectiveness, acceptability and conceptual basis. Aging and Mental Health. 1-18

Morris, L., Mansell, W., Williamson, T., Wray, A. \& McEvoy, P. (2018). Communication Empowerment Framework: An integrative framework to support effective communication and interaction between carers and people living with dementia. Dementia. doi: 10.1177/1471301218805329

Mittelman, M.S., Ferris, S.H., Shulman, E., Steinberg, G. and Levin, B., (1996) A family intervention to delay nursing home placement of patients with Alzheimer disease: a randomized controlled trial. Jama, 276(21), pp.1725-1731.

Mittelman, M.S., Roth, D.L., Coon, D.W. and Haley, W.E., (2004) Sustained benefit of supportive intervention for depressive symptoms in caregivers of patients with Alzheimer's disease. American Journal of Psychiatry, 161(5), pp.850-856. 
O'Connor, M.L. and McFadden, S.H., (2010). Development and psychometric validation of the Dementia Attitudes Scale. International Journal of Alzheimer's Disease, 2010.

O'Dwyer, S.T., Moyle, W., Zimmer-Gembeck, M. and De Leo, D., (2016). Suicidal ideation in family carers of people with dementia. Aging \& mental health, 20(2), pp.222-230.

O'dwyer, S.T., Moyle, W., Zimmer-Gembeck, M. and De Leo, D., (2013). Suicidal ideation in family carers of people with dementia: a pilot study. International journal of geriatric psychiatry, 28(11), pp.1182-1188.

Peräkylä, A. and Weiste, E. (2013) A comparative conversation analytic study of formulations in psychoanalysis and cognitive therapy. Research on Language and Social Interaction 46 (4): 299-321.

Pocklington, C., Gilbody, S., Manea, L. and McMillan, D., (2016). The diagnostic accuracy of brief versions of the

Geriatric Depression Scale: a systematic review and meta-analysis. International journal of geriatric psychiatry,

Plug, L., Sharrack, B. and Reuber, M., (2009). Conversation analysis can help to distinguish between epilepsy and non-epileptic seizure disorders: a case comparison. Seizure, 18(1), pp.43-50.

Robertson, S.M., Zarit, S.H., Duncan, L.G., Rovine, M.J. and Femia, E.E., (2007). Family caregivers' patterns of positive and negative affect. Family Relations, 56(1), pp.12-23.

Schreiner, A.S., Morimoto, T., Arai, Y. and Zarit, S., (2006). Assessing family caregiver's mental health using a statistically derived cut-off score for the Zarit Burden Interview. Aging and Mental Health, 10(2), pp.107-111.

Schulz, R. and Martire, L.M., (2004). Family caregiving of persons with dementia: prevalence, health effects, and support strategies. The American journal of geriatric psychiatry, 12(3), pp.240-249.

Shaw, C., Chrysikou, V., Lanceley, A., Lo, C., Hales, S. and Rodin, G., (2018). Mentalization in CALM psychotherapy sessions: Helping patients engage with alternative perspectives at the end of life. Patient Education and Counselling.

Jefferson, G (1989) Preliminary notes on a possible metric which provides for a 'standard maximum' silence of approximately one second in conversation. In D. Roger and P. Bull (eds.) Conversation: An Interdisciplinary Perspective 166-196. Clevedon: Multilingual Matters. 
Keselman, H., Osvaldsson Cromdal, K., Kullgard, N. and Holmqvist, R., (2018). Responding to mentalization invitations in psychotherapy sessions - A conversation analysis approach. Psychotherapy Research 28(4), pp.654-666.

Kramer, B.J., Boelk, A.Z. and Auer, C., (2006). Family conflict at the end of life: Lessons learned in a model program for vulnerable older adults. Journal of Palliative Medicine, 9(3), pp.791-801.

Kurtis L \& Netten A (2012) Unit costs of health and social care. University of Kent At Can.

Wenger, G.C., 1997. Social networks and the prediction of elderly people at risk. Aging \& Mental Health, 1(4), pp.311-320.

Wynn, R. and Wynn, M., 2006. Empathy as an interactionally achieved phenomenon in psychotherapy: Characteristics of some conversational resources. Journal of Pragmatics, 38(9), pp.1385-1397

Zoom Video Communications., 2018. https://www.zoom.us/ (accessed, 09/02/2019) 